\title{
Bandwidth enhancement of Fabry-Perot quantum-well lasers by injection-locking
}

\author{
Xiaomin Jin Shun-Lien Chuang \\ Electrical Engineering Department, California Polytechnic State University, San Luis Obispo, CA 93407, USA \\ Department of Electrical and Computer Engineering, University of Illinois at Urbana-Champaign, 1406 W. Green Street, Urbana, IL 61801, USA
}

\begin{abstract}
Theory and experiment for dc and small-signal electrical modulation of an injection-locked quantum-well (QW) Fabry-Perot laser are presented. Our experiment is realized by performing side-mode injection locking of a multiple-quantum-well (MQW) InGaAsP FabryPerot (FP) laser, which has the advantage of optical wavelength conversion. We first measure the dc characteristics and optical spectra of an injection-locked laser to define its locking range and linewidth enhancement factor. We then show experimentally that the bandwidth of an injection-locked semiconductor laser is $10.5 \mathrm{GHz}$, which is around twice the free-running electrical modulation bandwidth $(5.3 \mathrm{GHz})$. The relaxation frequency of the injection-locked laser can be 3.5 times greater than the free-running value. Our theoretical model includes mode competition, gain saturation, low frequency roll-off, and optical confinement factor of the QW structure. The theory shows good agreement with our experimental results. We point out that the small-signal modulation of injection-locked lasers still suffers severely from low frequency roll-off, which comes from the carrier transport effect and parasitic effect of the bias circuit. If we can reduce those effects, the modulation bandwidth can be further increased to $15 \mathrm{GHz}$, which is around 3 times of the free-running value.
\end{abstract}

Keywords: Optical injection; Injection locking; Modulation bandwidth; Semiconductor laser

\section{Introduction}

Recently, an intensive work has been conducted to investigate the injection locking in photonic devices and systems. This is because that by using the injection-locking technique, variety of ultra-fast optical components or systems can be designed, such as simultaneous repolarization polarization-scrambled wavelength channels [1], error-free detection of OC-192 DPSK signal [2], all-optical modulation format conversion and multicasting [3], optical frequency modulation and intensity modulation suppression [4], injection locking of VCSELs [5], 35-GHz intrinsic bandwidth for direct modulation in $1.3 \mu \mathrm{m}$ semiconductor lasers [6], an all-optical switch using a multi-wavelength mutual injection-locked laser diode [7], MEMs injectionlocked laser [8], and all-optical packet demultiplexing using a multi-wavelength mutual injection-locked laser diode [9].

An injection-locked laser system contains two semiconductor lasers. The light from a pump laser is injected into the test laser oscillating above threshold, and the injected radiation competes with the spontaneous emission of the test laser being amplified. If the optical frequency of the injected light is close to the eigenfrequency of the unperturbed laser, the test laser will adjust its frequency and coherence properties to that of the injected light. When a complete locked state is reached, all of the power of the test laser is emitted at the optical frequency of the master laser. This phenomenon is known as injection locking. Injection locking is a complex phenomenon because of 
the competition between the amplified spontaneous emissions and the amplified pump laser signal as well as the beating of these two fields. The change in gain by injection leads to a change of refractive index with carrier density. The inclusion of the gain change mechanism increases the complexity of laser behavior.

The electrical modulation of injection-locked lasers is one of the hottest topic and attracted considerable attention $[5,6,10]$. Because it is predicted that the modulation bandwidth of strongly injection-locked semiconductor lasers can be significantly improved compared to free-running electrical modulation [11,12]. The modulation bandwidth can be $2-3$ times of the free-running value. This is very attractive since it may allow one to achieve large modulation bandwidths with conventional semiconductor lasers at room temperature, avoiding the use of advanced devices and the need for complicated fabrication techniques. Furthermore injection locking in semiconductor lasers is an attractive method to ensure single mode operation [13], reduce the linewidth of a free-running laser [14], and eliminate mode partition noise [15], mode hopping, and frequency chirp from modulated lasers [16]. The injectionlocking technique may also prevent spurious feedback effects that are random and difficult to avoid and can strongly disturb the behavior of the laser. Furthermore, injection locking in semiconductor lasers is a promising method to generate microwave signals and synchronize one or more free-running lasers to a pump laser [17].

In this study, we report experiment results and theoretical calculations of the small-signal modulations of a sidemode injection-locked Fabry-Perot (FP) laser. Our test laser is a compressively-strained multi-quantum-well (MQW) InGaAsP semiconductor laser. The side-mode injection locking of FP lasers has the advantage of multimode selection over that of a single mode DFB laser, which is useful for wavelength division multiplexing (WDM) channel selection. In this way, the modulation signal at free-running frequency can be switched optically to different wavelength channels [18]. We first measure the dc characteristics of the injection-locked laser and obtain the linewidth enhancement factor of the test laser, which is in agreement with a measurement using an independent method based on amplified spontaneous emission spectroscopy [19]. We show that by injection locking the chirp is reduced and the side-mode suppression ratio of the FP laser improves. We also show the improvement of $3 \mathrm{~dB}$ modulation bandwidth of an injection-locked FP laser, which is twice of its free-running value. The relaxation frequency is 3.5 times of its free-running value. We also point out that the small-signal modulation of injection-locked lasers still suffers low frequency roll-off, which comes from the carrier transport effect and parasitic effect of the bias circuit. We improve the existing small-signal model for injection locking by adding the optical confinement factor of separate-confinement-heterostructure $(\mathrm{SCH}) \mathrm{QW}$ lasers, nonlinear gain saturation of the test laser due to the master laser, and low frequency roll-off due to carrier transport and parasitic effects. Our model includes all relevant phenomena, either observed experimentally or predicted theoretically in weak to moderate injection regimes and shows good agreement with our experimental results. This paper is organized as follows: in Section 2, the theory of dc and the small-signal electrical modulation of injection-locked lasers is presented. In Section 3, the experimental setup is described, and experimental results are shown and compared with theory. The conclusion is presented in Section 4.

\section{Theory for electrical modulation of injection-locked quantum-well lasers}

We consider two semiconductor lasers with a small difference in resonant wavelength (or frequency). The singlemode pump laser injects light into the other laser, referred to as the test laser. An optical isolator blocks the reverse light path. The locking occurs within a certain frequency locking range defined by the injection level and the amplitude-phase coupling coefficient. It is assumed that the effects of spatial hole burning in a FP laser are negligible. The theoretical analysis describing the injection-locked test laser is based on the rate equations, shown as below. We also need to consider the possible excitation of the neighboring longitudinal mode with different injections. This can be accounted for by a rate equation for the photon density $S_{\mathrm{u}}$ in the side modes or the group of side modes, which represents the unlocked photon density. Therefore, the total photon density is $S=S_{\mathrm{u}}+S_{1}$, where $S_{1}$ is the photon density in the locked mode.

$$
\begin{aligned}
& \frac{\mathrm{d} S_{1}(t) \longleftarrow}{\mathrm{d} t} \leftrightarrows \Gamma_{1} v_{\mathrm{g}} S_{\mathrm{l}}(t) G_{1}\left[1-\varepsilon_{\mathrm{l}} S_{\mathrm{l}}(t)-\varepsilon_{\mathrm{u}} S_{\mathrm{u}}(t)-\varepsilon_{\mathrm{i}} S_{\mathrm{i}}\right] \leftarrow \\
& -\frac{S_{1}}{\tau_{\mathrm{pl}}}+2 k_{\mathrm{c}} \sqrt{S_{1}(t) \phi_{\mathrm{i}}} \cos \left(\phi_{1}(t)-\phi_{\mathrm{i}}\right) \leftarrow \\
& \frac{\mathrm{d} S_{\mathrm{u}}(t) \leftarrow}{\mathrm{d} t}=\Gamma_{\mathrm{u}} v_{\mathrm{g}} S_{\mathrm{u}}(t) G_{\mathrm{u}}\left[1-\varepsilon_{\varepsilon_{1}} S_{1}(t)-\varepsilon_{\mathrm{u}} S_{\mathrm{u}}(t)-\varepsilon_{\mathrm{i}} S_{\mathrm{i}}\right] \leftarrow \\
& -\frac{S_{\mathrm{u}}(t) \leftarrow}{\tau_{\mathrm{pu}}} \\
& \frac{\mathrm{d} \phi_{l}(t)}{\mathrm{d} t}=-k_{\mathrm{c}} \sqrt{\frac{S_{\mathrm{i}}}{S_{\mathrm{1}}(t)}}\left(\left(\sin \left(\phi_{1}(t)-\phi_{\mathrm{i}}\right)-\left(\omega_{\mathrm{i}}-\omega_{0}\right) \leftarrow\right.\right. \\
& +\frac{\alpha}{2} \Gamma_{1} v_{\mathrm{g}} g_{1}^{\prime}\left[N(t)-N_{0}\right]\left[1-\varepsilon_{1} S_{1}(t)-\varepsilon_{\mathrm{u}} S_{\mathrm{u}}(t)-\varepsilon_{\mathrm{i}} S_{\mathrm{i}}\right] \leftarrow \\
& \frac{\mathrm{d} N(t)}{\mathrm{d} t}=\frac{I(t)}{e V}-\stackrel{N(t)}{\tau_{\mathrm{n}}} \leftarrow \\
& -v_{\mathrm{g}} S_{1}(t) G_{1}\left[1-\varepsilon_{1} S_{1}(t)-\varepsilon_{\mathrm{u}} S_{\mathrm{u}}(t)-\varepsilon_{\mathrm{i}} S_{\mathrm{i}}\right] \leftarrow \\
& \left.-v_{\mathrm{g}} S_{\mathrm{u}}(t) G_{\mathrm{u}} 1-\varepsilon_{1} S_{1}(t)-\varepsilon_{\mathrm{u}} S_{\mathrm{u}}(t)-\varepsilon_{\mathrm{i}} S_{\mathrm{i}}\right]
\end{aligned}
$$

where $S_{\mathrm{i}}$ is injected photon density into the test laser cavity from the master laser, $\phi_{1}(t)$ and $\phi_{\mathrm{i}}(t)$ are the phase of the locked mode and injected laser field, $N(t)$ is the carrier density, $\omega_{\mathrm{i}}$ is the master laser frequency, $\omega_{0}$ is the cavity resonance frequency of the test laser, $\alpha$ is the linewidth enhancement factor, $k_{\mathrm{c}}=\frac{c}{2 n_{\mathrm{g}} L}$ is the coupling coefficient, $n_{\mathrm{g}}$ is the group index of the test laser, $L$ is the test laser cavity length, the $I(t)$ is the test laser current, $V$ is the 
volume of the active region, $e$ is the unit charge of the carrier, $\tau_{\mathrm{n}}$ is the carrier lifetime, $v_{\mathrm{g}}$ is the group velocity, $\tau_{\mathrm{pl}}$ and $\tau_{\mathrm{pu}}$ are the photon lifetime for locked mode and unlocked mode, and $G_{1}=g_{10}+g_{1}^{\prime}\left(N(t)-N_{0}\right)$ and $G_{\mathrm{u}}=g_{\mathrm{u} 0}+\leftarrow$ $g_{\mathrm{u}}^{\prime \prime}\left(N(t)-N_{0}\right)$ are the gain of the locked and unlocked laser, and $g_{1}^{\prime \leftarrow}$ and $g_{\mathrm{u}}^{\prime \leftarrow}$ are the differential gain. For the smallsignal analysis, the quantity $N(t)-N_{0}$ will equal the small-signal change in carrier density, denoted by $n$. The spontaneous emission term has been neglected because the test laser is biased above threshold. We also include both nonlinear gain saturation coefficients, $\varepsilon_{1}$ and $\varepsilon_{\mathrm{u}}$, as an improvement of a previous model $[13,20]$ for injection locking in semiconductor QW lasers. Our contributions are listed bellow. First, we include, the optical confinement factor $\Gamma$, which is an important parameter for SCH QW laser structures, in the rate equations. The factor is well-known to be important for separate confinement quantum-well structures, however, it is usually ignored in the literature on injection locking. Second, we include the nonlinear gain saturation of the test laser due to the injected photon density $\varepsilon_{\mathrm{i}}$ and all optical modes in the cavity, which has been used in earlier studies [19,21] on high-speed lasers where the gain of the test laser light is suppressed due to the presence for the injected light. This is an important phenomenon if we inject light in the laser gain region and it cannot be neglected. As we will show later, inclusion of $I$ and $\varepsilon_{\mathrm{i}}$ is important to extra consistent parameters for the gain and differential gain for quantum-well lasers.

If no light is injected into the test laser (free-running $S_{\mathrm{i}}=0$ ), we can see from Eqs. (1)-(4) that only the photon density and carrier density are coupled. The phase term is not necessary for solving the photon density and carrier density. However in an injection-locked laser, whose injected photon density $S_{\mathrm{i}}$ is non-zero, there is an additional coupling of the magnitude and phase of photon field and carrier density through externally injected light from the master laser. This is a unique and important characteristic of injection-locked semiconductor lasers called mutual amplitude and phase coupling.

By solving for the steady-state solution of the rate equations, we obtain the expression of the locking bandwidth, which is given as a function of the injection rate and the phase difference $\Delta \phi=\phi-\phi_{\mathrm{i}}[22]$

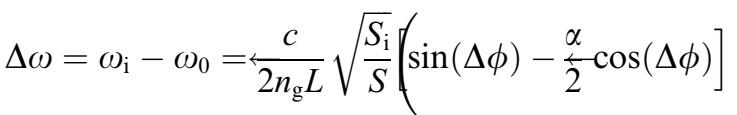

There are two ways by which a laser field oscillating at cavity resonance frequency $\omega_{0}$ in the absence of injection can be forced instead to oscillate at the frequency of the master laser $\omega_{\mathrm{i}}$. The first mechanism, which appears in every locking system, is to have the injected field add an out-of-phase component to the lasing mode of the test laser. This alters the phase of the test laser field and changes the frequency of the test laser field. The first term on the right-hand side of Eq. (5) represents this frequency shift. The second way to have the test laser field oscillate at $\omega_{\mathrm{i}}$ is to alter the cavity resonance frequency $\omega_{0}$ by the injected field changing the gain required to maintain the steady-state test laser intensity. The change in gain is accompanied by a shift in cavity resonance frequency. This is an extra term peculiar to semiconductor lasers arising from the refractive index dependence on carrier density. The second term of the expression (5) accounts for this cavity frequency shift due to the refractive index dependence on gain change mechanism [22], characterized by the linewidth enhancement factor $\alpha$.

The injection-locking range is determined by both the detuning $\left(\Delta \omega=\omega_{\mathrm{i}}-\omega_{0}\right)$ and the injection power. Using Eq. (5) and the condition that the injection locking is a constructive interaction with regard to the power balance, we obtain an asymmetric locking bandwidth [22]

$\Delta \omega_{\min }=-\frac{c}{2 n_{\mathrm{g}} L} \sqrt{\frac{S_{\mathrm{i}}}{S}\left(1+\left(\alpha^{2}\right)\right.}\left(<\Delta \omega<-\frac{c}{2 n_{\mathrm{g}} L} \sqrt{\frac{S_{\mathrm{i}}}{S}}=\Delta \omega_{\max }\right.$

Outside this region is the unlocked region, where the injection level is too low or the detuning is too high to reach locking condition. A detailed stability analysis can be found in [23], which also calculates a self-pulsation zone (a Hopf bifurcation), chaotic zone, and a coherence collapse zone. Here, we focus on the stable locking zone. We carefully control our experiment to make sure that the injected power is not strong enough to cause selfpulsation.

We also derive the small-signal modulation response of an injection-locked laser from rate equations, Eqs. (1)-(4). The changes in the lasing mode photon densities and carrier density due to small-signal modulation are assumed to be much smaller than the steady-state value of the photon and carrier densities. To solve for the small-signal modulation response, the expressions for carrier and photon densities are

$$
\begin{aligned}
& N(t)=N_{0}+n(\omega) \mathrm{e}^{j \omega t} \\
& S_{1}(t)=S_{10}+s_{1}(\omega) \mathrm{e}^{j \omega t} \\
& S_{\mathrm{u}}(t)=S_{\mathrm{u} 0}+S_{\mathrm{u}}(\omega) \mathrm{e}^{j \omega t} \\
& \phi_{1}(t)=\phi_{l 0}+\phi_{1}(\omega) \mathrm{e}^{j \omega t} \\
& I(t)=I_{0}+i(\omega) \mathrm{e}^{j \omega t}
\end{aligned}
$$

In our notation, capital letters stand for steady-state values. Taylor's series expansion is used to simplify the small-signal form of the rate equations. Terms containing products of the steady-state and small-signal components are linearized, and only the first-order terms are retained. The small-signal rate equations can be expressed as follows:

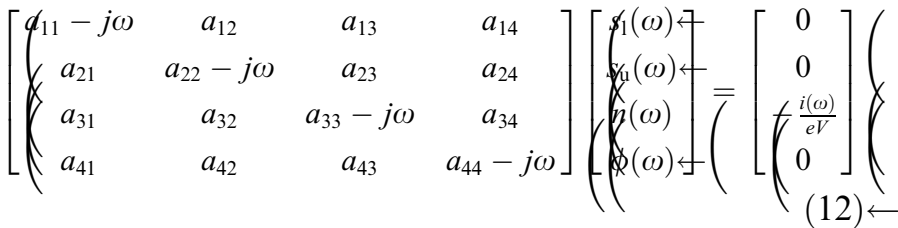


where

$$
\begin{aligned}
& a_{11}=-\Gamma_{1} v_{\mathrm{g}} g_{10}\left(2 \varepsilon_{1} S_{10}+\varepsilon_{\mathrm{u}} S_{\mathrm{u} 0}+\varepsilon_{\mathrm{i}} S_{\mathrm{i}}-1\right)-\frac{1}{\tau_{\mathrm{pl}}} \\
& +k_{\mathrm{c}} \sqrt{\frac{S_{\mathrm{i}}}{S_{10}}} \cos \left(\phi_{10}-\phi_{\mathrm{i}}\right) \\
& a_{12}=-\Gamma_{1} v_{\mathrm{g}} g_{10} \varepsilon_{\mathrm{u}} S_{10} \\
& a_{13}=\Gamma_{1} v_{\mathrm{g}} g_{1}^{\prime} S_{10}\left(1-\varepsilon_{1} S_{10}-\varepsilon_{\mathrm{u}} S_{\mathrm{u} 0}-\varepsilon_{\mathrm{i}} S_{\mathrm{i}}\right) \\
& a_{14}=-2 k_{\mathrm{c}} \sqrt{S_{1}\left(S_{\mathrm{i}}\right.} \sin \left(\phi_{10}-\phi_{\mathrm{i}}\right) \leftarrow \\
& a_{21}=-\Gamma_{\mathrm{u}} v_{\mathrm{g}} g_{\mathrm{u} 0} \mathrm{E}_{\mathrm{L}} S_{\mathrm{ul}} \\
& a_{22}=-\Gamma_{\mathrm{u}} v_{\mathrm{g}} g_{\mathrm{u} 0}\left(\varepsilon_{1} S_{10}+2 \varepsilon_{\mathrm{u}} S_{\mathrm{u} 0}+\varepsilon_{\mathrm{i}} S_{\mathrm{i}}-1\right)-\frac{1}{\tau_{\mathrm{pu}}} \\
& a_{23}=\Gamma_{\mathrm{u}} v_{\mathrm{g}} g_{\mathrm{u}}^{\prime} S_{\mathrm{u} 0}\left(1-\varepsilon_{1} S_{10}-\varepsilon_{\mathrm{u}} S_{\mathrm{u} 0}-\varepsilon_{\mathrm{i}} S_{\mathrm{i}}\right) \leftarrow \\
& a_{24}=0 \\
& a_{31}=v_{\mathrm{g}} g_{10}\left(2 \varepsilon_{1} S_{10}+\varepsilon_{\mathrm{u}} S_{\mathrm{u} 0}+\varepsilon_{\mathrm{i}} S_{\mathrm{i}}-1\right) \leftarrow \\
& +v_{\mathrm{g}} g_{\mathrm{u} 0} \varepsilon_{1} S_{\mathrm{u} 0} \\
& a_{32}=v_{\mathrm{g}} g_{\mathrm{u} 0}\left(\varepsilon_{1} S_{10}+2 \varepsilon_{\mathrm{u}} S_{\mathrm{u} 0}+\varepsilon_{\mathrm{i}} S_{\mathrm{i}}-1\right) \leftarrow \\
& +v_{\mathrm{g}} g_{10} \varepsilon_{\mathrm{u}} S_{10} \\
& a_{33}=-\frac{1}{\tau_{\mathrm{n}}}-v_{\mathrm{g}} g_{1}^{\prime} S_{10}\left(1-\varepsilon_{1} S_{10}-\varepsilon_{\mathrm{u}} S_{\mathrm{u} 0}-\varepsilon_{\mathrm{i}} S_{i}\right) \\
& -v_{\mathrm{g}} g_{\mathrm{u}}^{\prime} S_{\mathrm{u} 0}\left(1-\varepsilon_{1} S_{10}-\varepsilon_{\mathrm{u}} S_{\mathrm{u} 0}-\varepsilon_{\mathrm{i}} S_{\mathrm{i}}\right) \leftarrow \\
& a_{34}=0 \\
& a_{41}=\frac{k_{\mathrm{c}}}{2 S_{10}} \sqrt{\frac{\$_{\mathrm{i}}}{\$_{10}}} \sin \left(\phi_{10}-\phi_{\mathrm{i}}\right) \leftarrow \\
& a_{42}=0 \\
& a_{43}=\frac{\alpha}{2} \Gamma_{1} v_{\mathrm{g}} g_{1}^{\prime}\left(1-\varepsilon_{1} S_{10}-\varepsilon_{\mathrm{u}} S_{\mathrm{u} 0}-\varepsilon_{\mathrm{i}} S_{\mathrm{i}}\right) \leftarrow \\
& a_{44}=-k_{\mathrm{c}} \sqrt{\frac{S_{\mathrm{i}}}{S_{10}}} \cos \left(\phi_{10}-\phi_{\mathrm{i}}\right)
\end{aligned}
$$

After eliminating the carrier density $n$, the phase term $\phi_{1}$ and solving for $S=S_{1}\left(\omega+S_{\mathrm{u}}(\omega)\right.$, the intrinsic modulation response is obtained $\left|M_{\text {int }}(\omega)\right|^{2}=\left|\left[S_{1}(\omega)+S_{\mathrm{u}}(\omega)\right] / i(\omega)\right|^{2}$. It is very important to note that the electrical modulation is an extrinsic modulation, which includes parasitic and transport effects. Parasitic effects come from the bias circuit and the shunting of modulation current around the active layer, which will cause a low frequency roll-off of modulation response. At the same time, the carriers are injected from the outer edge of $\mathrm{SCH}$ region. The injected carriers diffuse through the $\mathrm{SCH}$ region and are captured into the quantum wells before recombining by stimulated emission processes. In the separate confinement structure QW lasers, the carrier diffusion, capture, and escape into and from QWs are usually defined to characterize the carrier transport processes [23] which give a parasitic-like roll-off and are indistinguishable from parasitic effects. In quantumwell lasers, the carrier transport time is an important limit for MQW laser modulation bandwidth. To complete the theory, the final modulation response of injection-locked lasers is

$$
\begin{aligned}
|M(\omega)|^{2} & =\frac{1}{1+\left(\omega / \omega_{\mathrm{p}}\right)^{2}}\left|M_{\mathrm{int}}(\omega)\right|^{2} \\
& =\frac{1}{1+\left(\omega / \omega_{\mathrm{p}}\right)^{2}}\left|\frac{S_{1}(\omega)+S_{\mathrm{u}}(\omega)}{(i(\omega) \leftarrow}\right|^{2}
\end{aligned}
$$

where $\omega_{\mathrm{p}}=2 \pi f_{\mathrm{p}}$ is the $16 \mathrm{w}$ frequency roll-off due to the transport effect and parasitic effect.

\section{Experimental setup and results}

\subsection{Experimental setup}

Electrical modulation of an injection-locked laser is performed on an InGaAsP Fabry-Perot laser, which is used as the test laser. The test laser threshold is $16 \mathrm{~mA}$ at $25^{\circ} \mathrm{C}$, and its active region has seven $-0.9 \%$ compressivelystrained quantum wells with an $80 \AA$ well width. The detailed composition of the undoped active region is described in previous work as sample A [24]. The experimental setup for small-signal modulation of injectionlocked lasers is shown in Fig. 1. The dc injection signal from a tunable pump laser passes through an erbiumdoped fiber optical amplifier (EDFA) which is used to control the injection power level. A tunable 3-nm bandwidth optical filter is used to remove excess signals on the side modes. The injection level is monitored by an optical power meter through an 1-99\% optical coupler. The HP 8510 network analyzer provides a small signal at frequencies swept from $500 \mathrm{MHz}$ to $18 \mathrm{GHz}$, which is coupled to the test laser electrodes through a high speed probe. The small-signal response is converted to an electrical signal using a photodetector and is increased by an $18 \mathrm{~dB}$-gain RF amplifier before entering the network analyzer, which measures the magnitude of the modulation response $|M(\omega)|^{2}$. The data has been averaged to reduce noise. An optical isolator is used to prevent feedback. An optical spectrum analyzer (OSA) is also used to measure the optical spectra of the test laser and master laser.

\subsection{DC analysis}

We first measure the lasing spectra of the pump laser and the injection-locked quantum-well test laser. Fig. 2 is the optical power spectrum of (a) the pump laser with $3.96 \mathrm{~mW}$ output power, (b) the free-running (dashed line) and injection-locked (solid line) test laser at $25 \mathrm{~mA}$ bias, and (c) the free-running (dashed line) and unlocked (solid line) test laser at $80 \mathrm{~mA}$ bias. The injection power in this paper represents the pump laser power measured before being coupled into the test laser, which is used as a standard for comparison. Fig. 2(a) shows that the pump laser is a tunable laser source emitting at $1557.45 \mathrm{~nm}$, which is close to the fifth side-mode on the longer wavelength side of the lasing mode of the test laser biased at $25 \mathrm{~mA}$. In 


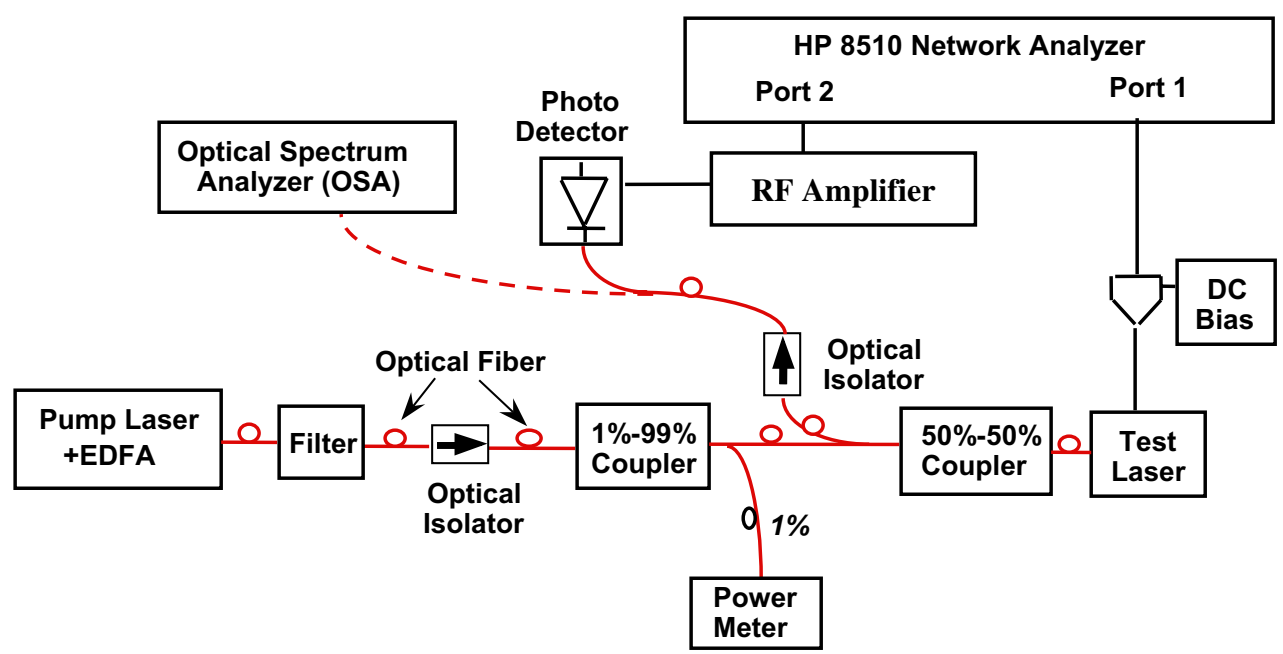

Fig. 1. The experimental setup for the small-signal modulation of injection-locked semiconductor lasers. The master laser is a tunable laser source. The test laser is an InGaAsP quantum-well laser, which can be directly modulated by current.

Fig. 2(b), the dashed line shows the laser emission spectrum of the test laser (biased at $25 \mathrm{~mA}, 25^{\circ} \mathrm{C}$ ) with the main peak wavelength at $1551.2 \mathrm{~nm}$. This is a typical FP laser spectrum, and the side-mode suppression ratio (SMSR) of the laser is around $20 \mathrm{~dB}$. When the external pump laser light is injected into the test laser biased above threshold, the injected light competes with the spontaneous emission of the laser for amplification. When the external light is strong enough and close to the eigenfrequency of the test laser, it is amplified since there is gain available. At the same time it saturates the gain of the other modes and extinguishes all the other free-running modes. Once a perfect locking state is reached, all of the power of the test laser is emitted at the optical frequency of the pump laser, as shown by the solid line in Fig. 2(b). Injection locking greatly improves the SMSR of the test laser and shifts the lasing wavelength from 1551.2 to $1557.45 \mathrm{~nm}$. This side-mode injection locking of a FP laser has the advantage of mode selection over that of a single-mode DFB laser. This inter-modal injection locking can switch the information from the free-running mode to any side mode as long as the injection-locking condition is satisfied, and can be used for optical wavelength conversion in wavelength division multiplexing (WDM) channel selection. In this way, the modulation signal at the free-running lasing wavelength can be switched optically to different wavelength channels [18]. When we increase the dc bias of the test laser, the wavelength of the test laser shifts from $1557.28 \mathrm{~nm}$ $(25 \mathrm{~mA})$ to $1557.75 \mathrm{~nm}(80 \mathrm{~mA})$. The detuning between the two lasers changes from $-20.89 \mathrm{GHz}$ to $+37.08 \mathrm{GHz}$, which changes the required power for injection locking. For example, if we inject the same injection signal into the test laser biased at a higher current of $80 \mathrm{~mA}$, the injected power is not strong enough to lock the test laser. We obtain unlocked test laser spectrum under injection, shown as the solid line in Fig. 2(c). The test laser is in multimode operation. We can also see the four-wave mixing peaks at several side modes. The resonant frequencies are not shifted by the external signal. The inability to lock the test laser is mainly because the injected power is smaller than the minimum required locking power at that detuning. The side-mode suppression is only the result of injection locking, which is determined by the relative intensity of the injected signal and the test signal under locking condition.

In Fig. 3(a), the wavelength of the test laser output versus the test laser bias current with (circles) and without (crosses) a $2.79 \mathrm{~mW}$ constant injection power is shown. Without injection, the test laser wavelength mode-hops as a function of current and is detrimental for modulation. The frequency chirp is also an important drawback under modulation. If we bias the test laser below $65 \mathrm{~mA}$ and injection lock the test laser, the output wavelength is fixed by the master laser regardless of its small-signal current variation. The electrical modulation of this injectionlocked laser system has the advantage of low chirp. Depending on the injection level and laser frequencies, the master field saturates gain more or less strongly. Therefore, it is not necessary that the two frequencies be close together to achieve synchronization, but then the locking may not be complete. This means that, if the injection level is not high enough to saturate the gain and to extinguish all the free-running modes, the energy is distributed among the free-running and locked modes. In the case, the locking is incomplete [22]. This is a unique characteristic of FP lasers. There is no incomplete locking in DFB lasers because the side mode suppression ratio of DFB lasers is much higher than FP lasers. If we change the test dc bias, we shift the oscillation frequency as well as increase the power of the test laser. A higher injection power is required to completely lock the laser. If we keep a constant injection level, we will start from a well-locked range to an incomplete locked range and finally to the unlocked regime. This is reflected in the test laser SMSR, which changes with the test laser bias, shown in Fig. 3(b). At low bias current (below $65 \mathrm{~mA}$ ), the SMSR is improved by injection locking. 

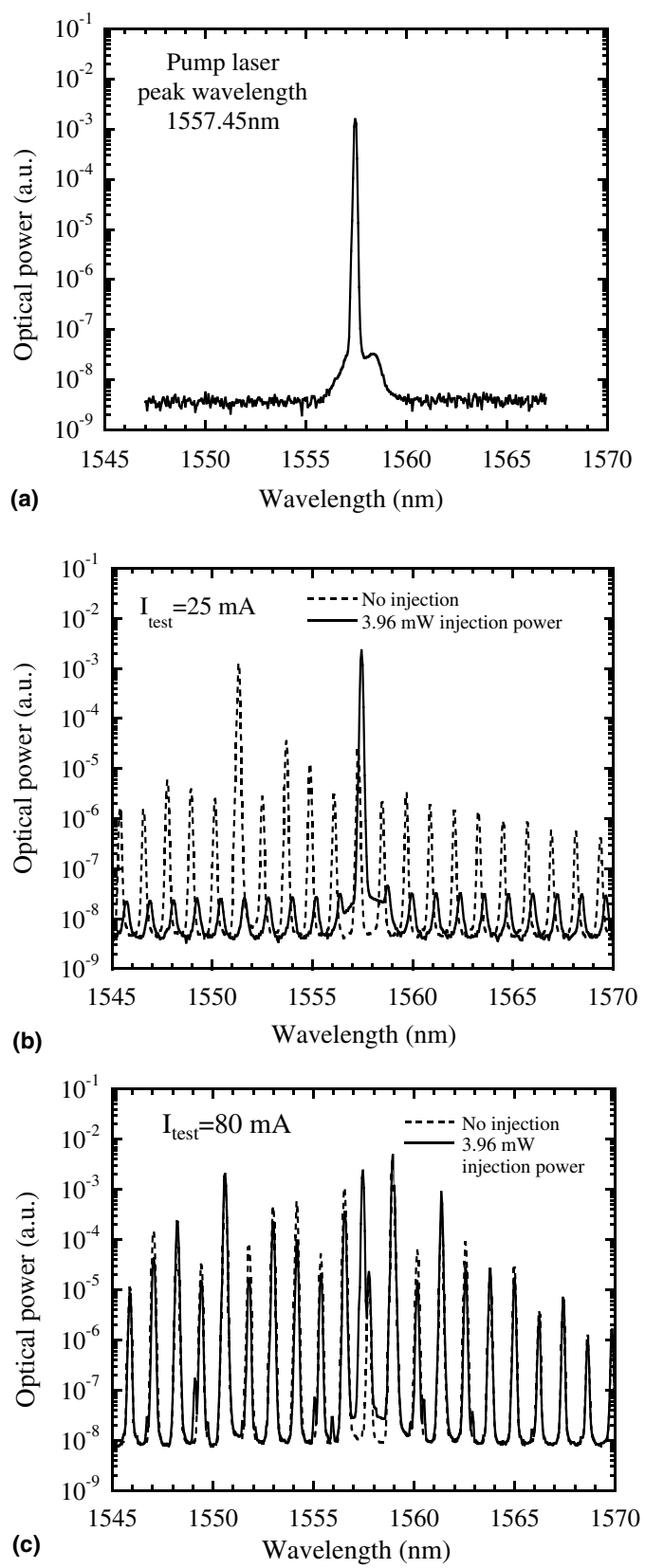

Fig. 2. Measured optical spectrum of (a) the test laser, (b) the test laser without injection (dashed line) and with injection (solid line) at $25 \mathrm{~mA}$ bias, (c) the test laser without injection (dashed line) and with injection (solid line) at $80 \mathrm{~mA}$ bias. The solid line in (b) is the injection-locked and (c) is unlocked test laser.

When the dc bias of the test laser increases, the SMSR decreases from $47 \mathrm{~dB}$ (locked) to $5 \mathrm{~dB}$ (unlocked). We can see a sudden $20 \mathrm{~dB}$ drop of SMSR between $65 \mathrm{~mA}$ and $70 \mathrm{~mA}$, which represents the boundary of locking and unlocking range. In summary, the overall locking range of a FP semiconductor laser is determined by both frequency detuning and the external injection power at a fixed test laser bias, shown in Fig. 3(c). From Eq. (5) and the two slopes at the boundaries of the locking range in Fig. 3(c), we can extract the linewidth enhancement factor of the test laser, $\alpha=\sqrt{\left.\left(\frac{\Delta \omega_{\min }}{\Delta \omega_{\max }}\right)\right)^{2}-1}(=4.8$, which is in agreement with
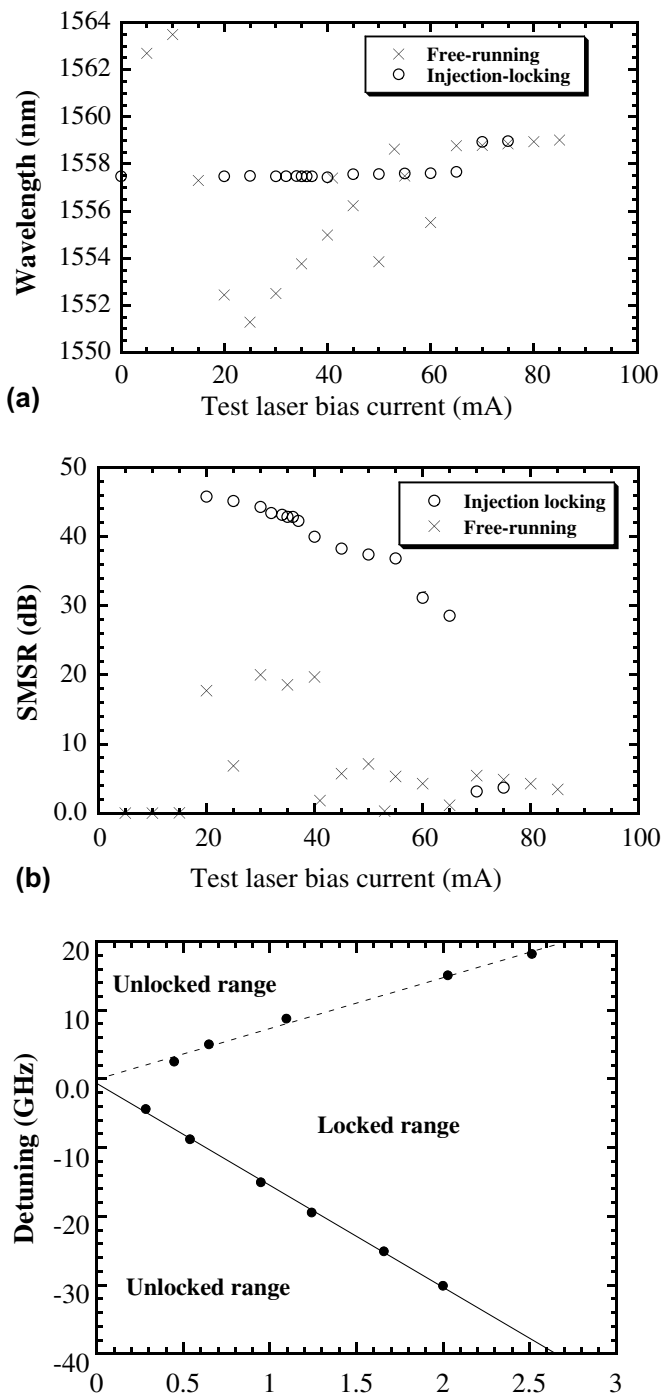

(c) Square root of minimum injection power $(\mathrm{mW})^{1 / 2}$

Fig. 3. Measured results for an injection-locked FP laser. (a) Test laser wavelength versus bias current. (b) Side-mode suppression ratio versus bias current for the free-running mode (crosses) and the $2.97 \mathrm{~mW}$ injection case (circles). (c) Locking range dependence on both injected power and detuning for a fixed test laser bias of $30 \mathrm{~mA}$ at $25^{\circ} \mathrm{C}$.

our previous independent measurement based on amplified spontaneous emission spectroscopy [19]. We will use this value in our theoretical calculation of the modulation response.

\subsection{Small-signal electrical modulation of injection-locked FP lasers}

The small-signal amplitude-modulation response of the injection-locked test signal is measured when a dc master laser signal is injected into the test laser biased above the threshold with small-signal modulation. Fig. 4 shows the modulation response of the injection-locked test laser under a constant test laser bias $I=30 \mathrm{~mA}$ at different injection powers $(0,0.34,0.65,1.32$, and $2.79 \mathrm{~mW})$. The power 
of the master laser is measured before it is injected into the test laser. Our small-signal modulation data was taken at different times from the dc data; thus, the coupling of the injected power into the test laser cavity is slightly different from that of the dc experiment. At $30 \mathrm{~mA}$ laser bias, we measure the optical spectra of the pump laser and the test laser. The frequency detuning is calculated from the difference of the lasing wavelength, which is $-15.3 \mathrm{GHz}$ and will be used for our modulation response calculation. The pump laser is offset at negative frequencies with respect to the free-running test laser to realize a stable locked configuration of the test laser at higher injection levels. The $3 \mathrm{~dB}$ bandwidth of injection-locked laser is $10.5 \mathrm{GHz}$ at $30 \mathrm{~mA}$, which is twice the free-running laser value $(5.3 \mathrm{GHz})$. Our data also show that the relaxation frequency of the free-running test laser at $30 \mathrm{~mA}$ bias current is $4 \mathrm{GHz}$. The relaxation frequency increases with increasing injection power and reaches $14 \mathrm{GHz}$ at an injection power around $2.79 \mathrm{~mW}$, which is about 3.5 times greater than the free-running value $(4 \mathrm{GHz})$. Also at higher laser bias (above $40 \mathrm{~mA}$ ), the free-running FP laser is in multimode operation, and its SMSR is below $10 \mathrm{~dB}$ as shown in Fig. 3(b). The injected signal reduces more unwanted fluctuations and feedback, more stimulated emission than random spontaneous emission occurs, and enhances the peaks. For an injection-locked FP laser, the total photon number in the test laser cavity increases very little under injection, which is only about several percent at high bias current. This means the improvement of relaxation frequency is not from the dramatic increase of the total power of the test laser. With external optical injection, the cavity gain is reduced to compensate the increase of the lasing mode photon density to realize the steady-state condition. The decrease in gain due to the optical injection brings about a decrease in the carrier number and spontaneous emission rate. For injection locking laser system, the photon density of the test laser is coupled mutually with its phase, which enhances the bandwidth. Note that this modulation method still cannot remove transport effects of QW structures and parasitic effect of the bias circuit. As shown

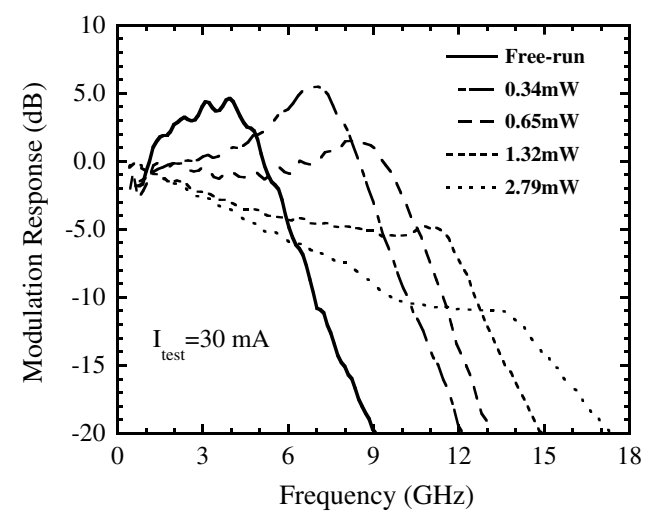

Fig. 4. Measured small-signal modulation response of the injection-locked test laser with a fixed test laser bias of $30 \mathrm{~mA}$ and injection powers of 0 , $0.34,0.65,1.32$, and $2.79 \mathrm{~mW}$. in Fig. 4, there is a severe drop-off of the modulation response at low frequencies. If the laser design can be improved to limit the transport and parasitic effects, injection locking can increase the modulation bandwidth to three times its free-running value.

To accurately model the modulation response, we include the low frequency roll-off $f_{\mathrm{p}}=7 \mathrm{GHz}$ into the intrinsic response, as shown in Eq. (13). The value of the low frequency roll-off is extracted by comparing the electrical modulation and optical absorption modulation of the test laser [25]. Our theoretical calculation results are shown in Fig. 5(a) for the test bias current of $30 \mathrm{~mA}$. We assume the total photon density in the test laser is constant $S_{0}=3.6 \times 10^{15} \mathrm{~cm}^{-3}$ for $30 \mathrm{~mA}$. The injected photon number varies from zero (free-running) to $S_{\mathrm{i}}=1.2 \times 10^{12} \mathrm{~cm}^{-3}$ and is linearly proportional to the injection power. The detunings used in the theoretical calculation are $-15 \mathrm{GHz}$. The gain saturation coefficients are fitting parameters $\varepsilon_{1}=\varepsilon_{\mathrm{i}}=\varepsilon_{\mathrm{u}}=2.02 \times 10^{17} \mathrm{~cm}^{-3}$. The optical confinement factor and the linewidth enhancement factor are obtained from previous experiments, which are 0.2 [24] and 1.8, respectively. The detailed parameters are listed in Table 1. Our calculated responses are in good agreement with
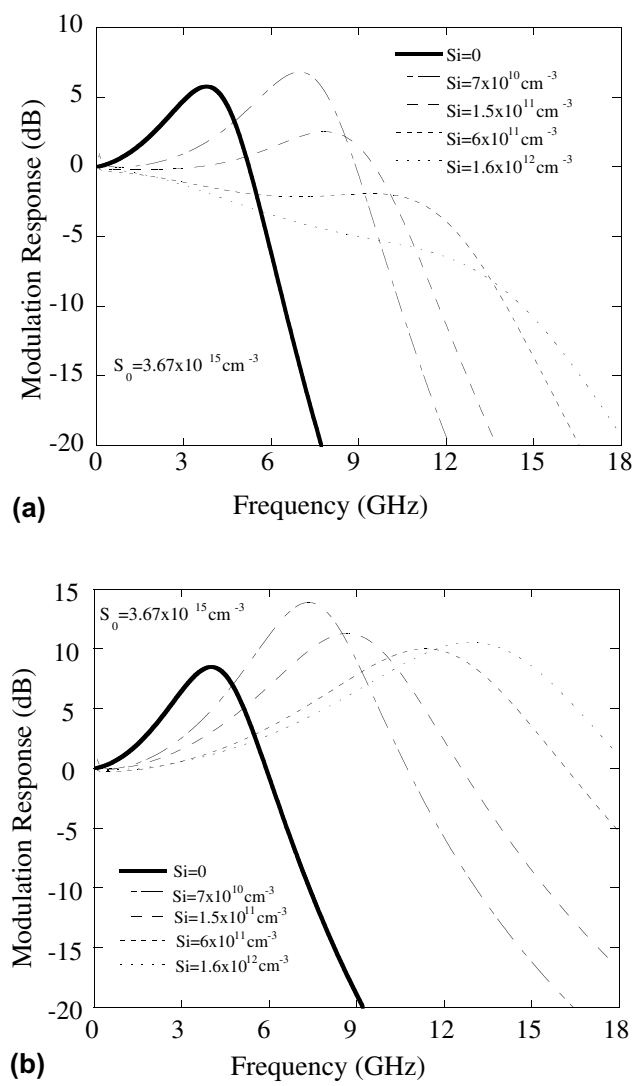

Fig. 5. Theoretical calculation of the small-signal modulation response of an injection-locked test laser with a fixed test laser photon density $S_{0}=3.6 \times 10^{15} \mathrm{~cm}^{-3}$ for $30 \mathrm{~mA}$ test laser bias. The injected photon number varies from zero (free-running) to $S_{\mathrm{i}}=1.2 \times 10^{12} \mathrm{~cm}^{-3}$ and is proportional to the injection power. (a) The low frequency roll-off is included with $f_{\mathrm{p}}=7 \mathrm{GHz}$. (b) The low frequency roll-off is not included with $f_{\mathrm{p}}=\infty \mathrm{GHz}$. 
Table 1

The test laser modeling parameters

\begin{tabular}{lll}
\hline Parameter & Symbols & Value \\
\hline Cavity length & $L$ & $290 \mu \mathrm{m}$ \\
Active volume & $V$ & $2.35 \times 10^{-11} \mathrm{~cm}^{3}$ \\
Effective index of refraction & $n_{\mathrm{g}}$ & 3.3 \\
Group velocity & $v_{\mathrm{g}}$ & $8.7 \times 10^{9} \mathrm{~cm} / \mathrm{s}$ \\
Mirror loss & $\alpha_{\mathrm{m}}$ & $43.15 \mathrm{~cm}^{-1}$ \\
Intrinsic loss & $\alpha_{\mathrm{i}}$ & $30 \mathrm{~cm}^{-1}$ \\
Optical confinement factor & $\Gamma$ & 0.2 \\
Linewidth enhancement factor & $\alpha$ & 1.8 \\
Photon lifetime & $\tau_{\mathrm{p}}$ & $5.8 \mathrm{ps}$ \\
Carrier lifetime & $\tau_{\mathrm{n}}$ & $0.8 \mathrm{~ns}$ \\
Differential gain & $g_{1}^{\prime \leftarrow}=\mathrm{g}_{\mathrm{u}}^{\prime \leftarrow}$ & $4 \times 10^{-16} \mathrm{~cm}^{2}$ \\
Nonlinear gain saturation coefficient & $\varepsilon_{\mathrm{l}}=\varepsilon_{\mathrm{u}}=\varepsilon_{\mathrm{i}}$ & $2.022 \times 10^{-17} \mathrm{~cm}^{3}$ \\
\hline
\end{tabular}

experimental data. The relaxation frequency increases by injection locking, and the relaxation peak value increases with increasing injection and reaches its maximum because of gain saturation effects. To understand the importance of the low frequency pole $f_{\mathrm{p}}$, we also calculate the intrinsic modulation response of the injection-locked laser without the low frequency roll-off, shown in Fig. 5(b) using the same parameters as Fig. 5(a). Fig. 5(b) is similar to previous theoretical paper on this subject [20] without low frequency rolloff. Our calculation results show that the low frequency pole limits modulation bandwidth, which is indicated clearly on our experimental data. The sharpness of the relaxation peak is dependent on the values of detuning, phase difference between the injected signal and test signal, and test laser photon density etc. Also our linear model is only sufficient to describe the stable dynamic domain. The relaxation frequency as a function of injection power is plotted in Fig. 6 . We convert the calculated injected photon density into the injected power $P_{\text {in }}$ using $P_{\text {in }}=2.6 \times 10^{-12} S_{\mathrm{i}} \mathrm{mW}$ to compare with our data. The solid circles are our experimental data at the test laser bias current of $30 \mathrm{~mA}$, and the dashed lines are the theoretical result. Starting at a weak injection (injection power $0.15 \mathrm{~mW}$ ), the relaxation oscillation frequency is

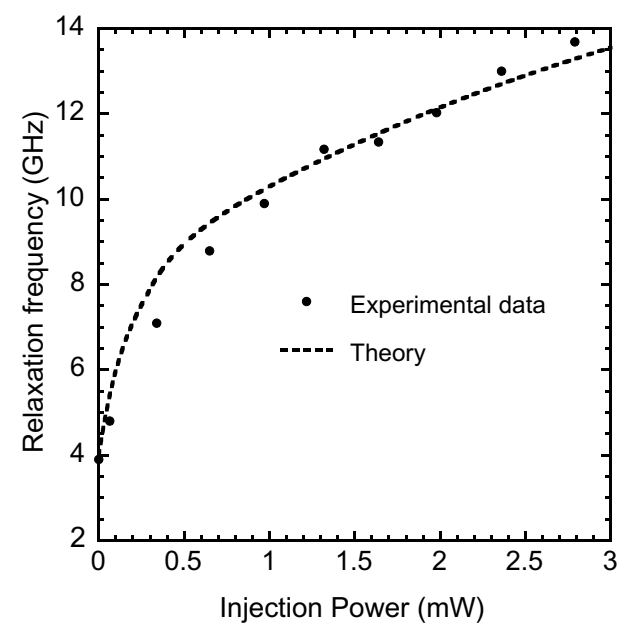

Fig. 6. The relaxation frequency versus the test laser bias. The symbols are results for experimental data, and the line is theoretical calculation. improved. The maximum relaxation frequency measured $(13.8 \mathrm{GHz})$ is about 3.5 times that of the free-running value $(4 \mathrm{GHz})$ at $30 \mathrm{~mA}$ laser bias. Our calculation shows very good agreement with our experimental data. In general, our model with low frequency roll-off represents a more complete picture of injection-locked semiconductor lasers and explains the experimental data very well. Note that our model also includes the optical confinement factor of the SCH QW structure and gain saturation from the injected signal, which are important because the value of differential gain would otherwise be incorrect for this system compared with our other independent measurement [24].

Several groups [12,20,26,27] have proposed possible mechanisms for the bandwidth enhancement by injection locking. They show bandwidth enhancement occurs under proper excitation conditions, for injection power and optical frequency detuning. The main reason for the bandwidth enhancement is the additional coupling of photon number to the phase of the light in the test laser for injection locked laser system, which enhances both the relaxation resonance frequency and the damping rate [12,20,27]. Our research also shows theoretically that the coherent addition of the injection optical field with the test laser optical field in the test laser cavity, rather than by the increase in photon density, is the main reason for improvement of bandwidth. Without any injected signal $S_{\mathrm{i}}$, the rate equation of the test laser amplitude is coupled to its phase only through the linewidth enhancement factor (see Eqs. (1)-(4)). The phase of the photon in the test laser has no feedback to the amplitude. And the phase term is not necessary when solving for amplitude modulation. In an injection-locked laser system, the injected photon term directly connects the amplitude and phase of the test laser. The phase term must be counted to obtain amplitude value. At certain phase condition, the injection signal will produce an extra term in amplitude rate equation, which results an additional term of $k_{\mathrm{c}} S_{\mathrm{i}}$ in relaxation frequency expression. These unique characteristics of the injection-locked laser system improve modulation bandwidth. Without any injection, the test laser behaves as a solitary laser. The additional terms in the relaxation resonance frequency of the injection locked laser system come from the phase-amplitude mutual coupling. The enhancement of the relaxation resonance frequency can also be attributed to the intensity of the injected field and the gain change (caused by nonlinear gain saturation terms), which is the second reason of bandwidth variation under injection. Generally, any change in the injection power or the gain will alter the relaxation resonance frequency. Furthermore, an important effect of external optical injection in the stable locking regime is to reduce the cavity gain due to a reduction in carrier density, which shifts the optical resonance frequency and eventually modify relaxation frequency and modulation bandwidth. The additional term in the relaxation frequency related to the nonlinear gain saturation can improve or reduce the modulation bandwidth. To accurate explain our data; the gain saturation term must be included. However, this is a smal- 
ler effect compared to the mutual amplitude-phase coupling. Finally, the nonlinear gain saturation term due to the pump laser, which represents the gain change caused by the pump laser injection, modifies the damping factor of the laser system.

\section{Conclusions}

We have theoretically and experimentally shown the enhancement of modulation bandwidth of an injectionlocked quantum-well FP laser. The enhancement of modulation bandwidth comes from the mutual amplitude and phase coupling of injection-locked semiconductor lasers. Rate equations for injection-locked lasers including the optical confinement factor of SCH QW structure have been presented. Our model explains the variation of the relaxation frequency with different injection power. Comparison between small-signal modulation of free-running lasers and injection-locked lasers is also presented, which shows the improvement of modulation bandwidth by injection locking. We show experimentally that the bandwidth of an injection-locked semiconductor laser is $10.5 \mathrm{GHz}$, which is around twice the free-running electrical modulation bandwidth $(5.3 \mathrm{GHz})$. The relaxation frequency of the injection-locked laser can be 3.5 times greater than the free-running laser. We point out that the small-signal modulation of injection-locked lasers still suffers severe low frequency roll-off, which comes from the carrier transport effect and parasitic effect of the bias circuit. If we can reduce these effects, the modulation bandwidth can be further increased to $15 \mathrm{GHz}$, or about three times the freerunning value.

\section{Acknowledgement}

We thank Dr. T. Tanbun-Ek for providing the quantum-well lasers for this study.

\section{References}

[1] Chan LY, Chung WH, Wai PKA, Moses B, Tam HY, Demokan MS. IEEE Photon Tech Lett 2002;14:1740.
[2] Awaji Y, Kuri T, Chujo W. In: Proceedings of CLEO '01, Technical digest 2001. p. 500.

[3] Chow CW, Wong CS, Tsang HK. J Lightwave Tech 2004;22:2386.

[4] Haldar MK, Coetzee JC, Gan KB. IEEE J Quantum Electron 2005;41:280.

[5] Chang C, Chrostowski L, Chang-Hasnain CJ. IEEE J Sel Top Quantum Electron 2003;9:1386.

[6] Hwang SK, Liu JM, White JK. IEEE Photon Tech Lett 2004;16:972.

[7] Nakkeeran K, Wai P.A, Chan LY, Lui LFK, Tam HY. In: Proceedings of the sixth chinese symposium optoelectronics, vol. 12. 2003. p. 175.

[8] Zhang XM, Liu AQ, Cai H, Lu C, Tang DY. In: The 13th international conference on solid-state sensors, 2005. Digest of technical papers, vol. 1. 2005. p. 388

[9] Chan LY, Wai PKA, Lui LFK, Moses B, Chung WH, Tam HY. OFC 2003;2:671.

[10] Lin GR, Lin YH, Chang YC. IEEE J Quantum Electron 2004; $40: 1014$

[11] Simpson TB, Liu JM. IEEE Photon Tech Lett 1997;9:1322.

[12] Wang J, Haldar MK, Lin L, Mendis FVC. IEEE Photon Tech Lett 1996;8:34

[13] Malyon DJ, McDonna AP. Electron Lett 1982;18:445.

[14] Gallion P, Nakajima H, Debarge G, Chabran C. IEEE J Quantum Electron 1985;QE-22:626.

[15] Jin X, Chuang SL. Appl Phys Lett 2000;77:1250.

[16] Noël L, Marcenac D, Wake D. Electron Lett 1996;25:1895.

[17] Laperle C, Svilans M, Poirier M, Têtu M. IEEE Trans Microwave Theory Tech 1999;47:1219.

[18] Hong Y, Shore KA, Lawrence JS, Kane DM. Appl Phys Lett 2000;76:3170

[19] Keating T, Park SH, Minch J, Chuang SL. Proc SPIE 1998;3283:314.

[20] Yabre G. IEEE J Quantum Electron 1996;14:2367.

[21] Vassilovski D, Wu TC, Kan S, Lau KY, Zah CE. IEEE Photon Tech Lett 1995;7:706.

[22] Petitbon I, Gallion P, Debarge G, Chabran C. IEEE J Quantum Electron 1988;24:148.

[23] Annovazzi-lodi V, Scire A, Sorel M, Donati S. IEEE J Quantum Electron 1998;34:2350

[24] Minch J, Park SH, Keating T, Chuang SL. IEEE J Quantum Electron 1999;35:1526.

[25] Keating T, Jin X, Chuang SL, Hess K. IEEE J Quantum Electron 1999;35:1526.

[26] Simpson TB, Liu JM, Gavrielides A. IEEE J Quantum Electron 1996;32:1456

[27] Lin L. IEEE J Quantum Electron 1994;30:1701. 